OPEN ACCESS

Edited by:

Anne Eckert,

University Psychiatric Clinic Basel,

Switzerland

Reviewed by:

Jie Lu,

Capital Medical University, China

Feng Liu,

Tianjin Medical University General

Hospital, China

*Correspondence:

Tianyou LuO

Itycqmu@163.com

Received: 24 August 2020 Accepted: 18 March 2021 Published: 12 May 2021

Citation:

Liu X, Cheng R, Chen L, Gong J, Luo T and LV F (2021) Altered Neurovascular Coupling in Subcortical Ischemic Vascular Disease.

Front. Aging Neurosci. 13:598365. doi: 10.3389/fnagi.2021.598365

\section{Altered Neurovascular Coupling in Subcortical Ischemic Vascular Disease}

\author{
Xiaoshuang Liu', Runtian Cheng ${ }^{1}$, Li Chen ${ }^{2}$, Junwei Gong ${ }^{1}$, Tianyou Luo ${ }^{1 *}$ and Fajin Lv' \\ ${ }^{1}$ Department of Radiology, First Affiliated Hospital of Chongqing Medical University, Chongqing, China, ${ }^{2}$ Department \\ of Radiology, Affiliated Hospital of North Sichuan Medical College, Sichuan, China
}

Patients with subcortical ischemic vascular disease (SIVD) exhibit a high risk of cognitive impairment that might be caused by neurologic deficits and vascular injuries. However, the mechanism remains unknown. In current study, 24 normal controls (NC) and 54 SIVD patients, including 26 SIVD patients with no cognitive impairment (SIVD-NCI) and 28 SIVD patients with mild cognitive impairment (SIVD-MCI) underwent the restingstate functional MRI (rs-fMRI) and neuropsychological assessments. We combined regional homogeneity (ReHo) and cerebral blood flow (CBF) by using the global ReHoCBF correlations coefficient and the ReHo/CBF ratio to detect the inner link between neuronal activity and vascular responses. Correlations between the $\mathrm{ReHo} / \mathrm{CBF}$ ratio and neuropsychological assessments were explored in patients with SIVD. As a result, we identified significantly decreased global ReHo-CBF coupling in the SIVD-NCI group and SIVD- $\mathrm{MCl}$ group with respect to the NC. The SIVD-MCl group showed more serious decoupling of the global ReHo-CBF correlation. We also found a significantly abnormal $\mathrm{ReHo/CBF}$ ratio predominantly located in cognitive-related brain regions, including the left insula, right middle temporal gyrus, right precuneus, left precentral gyrus, and left inferior parietal lobule but not the supramarginal and angular gyri. The SIVD-MCI group showed more severe disorders of neurovascular coupling than the other two groups. Moreover, the ReHo/CBF ratio in the left precentral gyrus of the SIVD-NCI group exhibited a positive correlation with the MMSE scores. These findings suggested that patients with SIVD show abnormal neurovascular coupling at the early stage of the disease and during disease development. It might be associated with disease severity and cognitive impairment. Neurovascular decoupling in brain may be a possible neuropathological mechanism of SIVD.

Keywords: subcortical ischemic vascular disease, neurovascular coupling, cerebral blood flow, functional magnetic resonance imaging, cognitive deficit, arterial spin labeling

\section{INTRODUCTION}

Subcortical ischemic vascular disease (SIVD) is a devastating illness with unclear etiology. It is a leading cause of subcortical vascular cognitive impairment (SVCI) (Rosenberg et al., 2014). Inapparent or even non-existent cognitive impairment was presented in the early stage of SIVD, and such individuals may suffer a high risk of future cognitive deficits (Sachdev et al., 2014). It is 
important to explore the pathological changes in the early stage of the disease. As the most common type of cerebral smallvessel disease (CSVD), SIVD may be related to the damaged endothelial cells, abnormal perfusion, and disrupted structural and functional connections in the brain (Wallin et al., 2018; Thrippleton et al., 2019). These alterations can promote the dysregulation of the neurovascular unit (NVU) which comprised neurons, astrocytes, and vessels (Muoio et al., 2014). It is acknowledged that the NVU plays a vital role in maintaining the homeostasis of the cerebral microenvironment to support the normal function in the brain (Pasley and Freeman, 2008; Helman and Murphy, 2016). Under physiological condition, microvascular blood flow matches well with the neurons and glia within the NVU, which is called neurovascular coupling (Girouard and Iadecola, 2006). CSVD could disturb this coupling and lead to incongruities between the cerebral blood supply and neuronal activity which might be treated as the main cause of cognitive impairment, but the mechanisms still cannot be fully explained (Caruso et al., 2019; Moretti and Caruso, 2020).

In recent decades, neuroimaging techniques have provided evidence for neural impairment (Xu et al., 2018; Qin et al., 2019; van Leijsen et al., 2019) and vascular impairment (Wardlaw et al., 2017; Wong et al., 2019; Zhang et al., 2019) in patients with CSVD. As expected, several studies have revealed a close correlation between brain activity and cerebral perfusion, and the results confirmed that the impairment of any component of the NVU can affect the neurovascular coupling associated with SVCI (Zlokovic, 2010; Murphy et al., 2016). The functional connectivity (FC) of brain regions is widely studied by restingstate functional MRI (rs-fMRI) through testing blood oxygen level-dependent (BOLD) signals (Hirano and Yamada, 2013; $\mathrm{Ma}$ et al., 2020). It has been suggested to reflect the essential functional relevance in the brain (Fox and Raichle, 2007). Decreased FC in patients with SIVD has been found in specific regions involved in cognitive functional organizations ( $\mathrm{Li}$ et al., 2014). Reduced efficiencies in cortical-subcortical circuits and disrupted pathways between distinct brain regions were found to be related to the cognitive dysfunctions (Reijmer et al., 2016; Liu et al., 2019b). Networks relevant to the cognitive function were also showed dysconnectivity in patients with SIVD (Liu et al., 2019a). Another analytical measure, the resting-state cerebral blood flow (CBF) is closely contacted with glucose utilization, oxygen consumption and aerobic glycolysis that maintaining cerebral activities (Vaishnavi et al., 2010). Arterial spin labeling (ASL) MRI is a non-invasive technique that can rapidly quantify CBF (Golay et al., 2004). Previous studies have revealed that the $\mathrm{FC}$ of brain regions showed similar patterns to the CBF (Liang et al., 2013; Zhu et al., 2013). Patients with SIVD suffer perfusion deficits accompanied by abnormal FC that may relate to the cognitive impairment (Sun et al., 2016).

However, these studies depended on only single imaging techniques that describe either the cerebral perfusion or neuronal activity, which could not comprehensively present the neurovascular coupling dysregulations in the disease. Neuronal activity and cerebral perfusion should be regarded as a functional complex. Combined BOLD and ASL technologies have been recently applied for studying neurovascular coupling because their correlations could be analyzed with the cooperativities of cerebral perfusion and neuronal activity of each voxel in the brain (Liang et al., 2013). Several studies combined the two parameters by evaluating their correlation coefficient and ratio to detect the alterations of NVU in diseases. The results have revealed the decoupling of NVU may be a crucial factor of the mechanisms related to cognitive impairment (Sheng et al., 2018; Hu et al., 2019; Stickland et al., 2019). It has been confirmed that CSVD could injure NVU and lead to SVCI (Shabir et al., 2018; Caruso et al., 2019). However, research rarely emphasizes the alterations of NVU in patients with SIVD by combining BOLD and ASL techniques.

The regional homogeneity ( $\mathrm{ReHo}$ ) is derived from BOLD signals that quantify the similarity of a given voxel to those of its nearest neighbors in a voxel-wise manner. Previous studies have shown that the ReHo of spontaneous activity is susceptible to SIVD which may correlate to the cognitive impairment (Liu et al., 2014; Diciotti et al., 2017). In addition, the CBF maps extracted from ASL signals are non-invasive measures that evaluate the cerebral perfusion. In the present study, we combined ReHo and CBF to detect the inner link between the neuronal activity and vascular response and its clinical significance in patients with SIVD. We hypothesized that patients with SIVD would show abnormal neurovascular coupling at the early stage of the disease and in disease development. The ReHo-CBF correlation coefficient measures the consistency of spatial distributions between cerebral blood supply and neuronal activity at the voxel level in whole brain, and the $\mathrm{ReHo} / \mathrm{CBF}$ ratio represents the connectivity strength between neurons and the surrounding brain areas that are supplied by a unit of CBF; these were used to estimate regional dysregulations of neurovascular coupling. We expect to provide additional information on the neuropathological mechanisms of SIVD.

\section{MATERIALS AND METHODS}

\section{Participants}

Seventy-eight participants were recruited for the study, including 24 normal controls (NC) and 54 patients with SIVD (26 patients with no cognitive impairment, SIVD-NCI; 28 patients with mild cognitive impairment, SIVD-MCI). Each subject signed an informed consent form approved by the ethics committee of first affiliated hospital of Chongqing medical university.

The criteria of patients with SIVD were listed as follows: (1) white matter hyperintensities: extended caps (>10 mm) or diffusely confluent hyperintensities with irregular shape ( $>25 \mathrm{~mm}$ ) intruded into the periventricular and deep white matter; and (2) lacunar cases: at least two lacunas in deep gray matter and accompanied by moderate white matter hyperintensities. The SIVD-MCI patients would be recruited if they met the criteria of SIVD accompanied by cognitive deficits, including the subjective cognitive complaints and objective cognitive impairments, but not satisfying the Diagnostic and Statistical Manual of Mental Disorders, fifth edition (DSMV). Meanwhile, the SIVD-MCI patients should also get the 
Clinical Dementia Rating Scale (CDR) scores of 0.5 and MiniMental State Examination (MMSE) scores ranging from 23 to 26. The SIVD-NCI patients were enrolled if they have met the diagnosis of SIVD with normal cognition and normal daily life abilities (defined as the CDR scores $=0$ and MMSE scores $\geq 27$ ). Exclusion criteria for all participants included (1) psychiatric disorders, such as schizophrenia or depression; (2) neurodegenerative disorder like Parkinson's disease; and (3) anyone with a metallic foreign body or other relevant magnetic resonance (MR) scanning contraindications.

\section{Neuropsychological Assessment}

All participants underwent the following neuropsychological assessments: (1) life abilities evaluation: Activities of Daily Living scale (ADL); (2) global cognition test: MMSE; (3) episodic memory test: Auditory Verbal Learning Test (AVLT); (4) language function test: Boston Naming Test (BNT); (5) visuospatial perception test: Clock Drawing Test (CDT); (6) executive functions and working memory assessment: Trail Making Test (TMT-A and TMT-B) and Stroop Test 1 and 2 (color-word); and (7) Hamilton Depression Scale (HAMD), which was used to exclude those with potential depression.

\section{MRI Acquisition}

MRI scanning was performed on a GE Signa Hdxt 3.0 T scanner. rs-fMRI data was acquired using an echo-planar imaging (EPI) pulse sequence: repetition time $(\mathrm{TR})=2,000 \mathrm{~ms}$; echo time $(\mathrm{TE})=40 \mathrm{~ms}$; flip angle $(\mathrm{FA})=90^{\circ}$; slice thickness $=4 \mathrm{~mm}$, no gap; field of view $(\mathrm{FOV})=240 \times 240 \mathrm{~mm}^{2}$; matrix $=64 \times 64$; and timepoints $=240$. A pseudo continuous ASL ( $\mathrm{pcASL}$ ) sequence with a $3 \mathrm{D}$ fast spin-echo acquisition and background suppression was applied for the perfusion imaging: $\mathrm{TR}=5,216 \mathrm{~ms}$; $\mathrm{TE}=9.8 \mathrm{~ms}$; spiral in readout of eight arms with 512 sample points; FOV $=240 \times 240 \mathrm{~mm}^{2}$; post-label delay $(\mathrm{PLD})=2,525 \mathrm{~ms}$; reconstruction matrix $=128 \times 128$; slice thickness $=4 \mathrm{~mm}$, no gap; and number of excitations $=3$. $3 \mathrm{D}-\mathrm{T} 1$ weighted images were scanned as follows: $\mathrm{TR}=8.3 \mathrm{~ms}$; $\mathrm{TE}=3.3 \mathrm{~ms}$; flip angle $=15^{\circ}$; thickness $=1 \mathrm{~mm}$, no gap; $\mathrm{FOV}=240 \times 240 \mathrm{~mm}^{2}$; matrix $=240 \times 240$, and voxel $=1 \times 1 \times 1 \mathrm{~mm}^{3}$. The scan parameters of the T2FLAIR weighted images were scanned as follows: $\mathrm{TR}=8,000 \mathrm{~ms}$; $\mathrm{TE}=26 \mathrm{~ms} ; \mathrm{TI}=1,500 \mathrm{~ms}$; thickness $=5 \mathrm{~mm}$, no gap; $\mathrm{FOV}=240 \times 240 \mathrm{~mm}^{2} ;$ and matrix $=256 \times 192$.

\section{Data Preprocessing}

fMRI data preprocessing was performed using the Data Processing Assistant for Resting-State fMRI (DPARSF) ${ }^{1}$ based on Statistical Parametric Mapping 8 (SPM8) ${ }^{2}$. The steps were listed as follows: (1) the first 10 timepoints were removed for each subject; (2) different acquisition times across slices were corrected by slice timing; (3) realignment for head motion was performed (head motion $>3.0 \mathrm{~mm}$ translation or $>3.0^{\circ}$ rotation were excluded). Instantaneous head motion of each volume was also evaluated by calculating the mean framewise

${ }^{1}$ http://rfmri.org/DPARSF

${ }^{2} \mathrm{http} / / /$ www.fil.ion.ucl.ac.uk/spm displacement (FD), and no significant differences among three groups were found in the mean FD; (4) the linear trends from the image time series were removed; (5) filtering with a frequency range of $0.01-0.08 \mathrm{~Hz}$ was performed; (6) spatial normalization into the standard Montreal Neurological Institute (MNI) space was performed by using EPI templates and resampled to $3 \times 3 \times 3 \mathrm{~mm}^{3}$ voxels; and (7) the nuisance covariates like global mean signal, cerebrospinal fluid signal, white matter signal, and 24 head motion parameters were regressed.

The CBF maps were generated with the software provided by the scanner vendor, and the SPM8 software was used to normalize the CBF images into MNI space. The preprocessing steps were specifically involved as follows: (1) the individual CBF maps were non-linearly co-registered to MNI space which generated a customized CBF template; (2) the individual CBF maps were non-linearly co-registered to the customized CBF template; (3) non-brain tissue were removed from each co-registered CBF map and normalized by the subject's global mean gray matter CBF value; and (4) the normalized CBF maps were smoothed by using a 6-mm full-width at half-maximum (FWHM) Gaussian kernel.

In addition, the white matter hyperintensity ( $\mathrm{WMH}$ ) volumes were assessed on the T2-FLAIR weighted images and 3D-T1 weighted images by AccuBrain ${ }^{\mathrm{TM}}$, a brain quantification tool that calculates the brain structure and $\mathrm{WMH}$ volume in a fully automatic mode.

\section{Data Analysis}

\section{ReHo Calculation}

ReHo data were computed by the DPARSF. Individual ReHo images were generated by calculating the Kendall's coefficient of concordance (KCC) between the time series of each voxel and those of its 26 neighboring voxels within a gray matter mask (Zang et al., 2004). Then, each individual's ReHo map was divided by its own global mean KCC value within the mask to achieve standardization. Finally, the standardized maps were then spatially smoothed with a $6 \mathrm{~mm} \times 6 \mathrm{~mm} \times 6 \mathrm{~mm}$ FWHM Gaussian kernel.

\section{Coupling Analysis}

Each subject underwent correlation coefficient analysis across voxels to quantitatively evaluate the consistency of spatial distributions between ReHo and CBF (Liang et al., 2013). The ratios of ReHo to CBF were computed by the DPARSF to evaluate the connectivity strength between neurons and the surrounding brain areas supplied by a unit of CBF. Meanwhile, the ratio of each voxel for each participant was transformed into a z-score to increase normality.

\section{Statistical Analysis}

Demographics, WMH volumes, and the scores of neuropsychological assessments were compared using SPSS 23.0 software. Normality for continuous variables was examined using Skewness-Kurtosis test. One-way ANOVA and post hoc comparisons were used to evaluate statistical differences. The chisquare test and the Kruskal-Wallis $H$ test were used to analyze the sex proportions and non-parametric data, respectively. 
In $\mathrm{ReHo}-\mathrm{CBF}$ correlation coefficient analysis and $\mathrm{ReHo} / \mathrm{CBF}$ ratio analysis, ANCOVA and post hoc individual tests were used to compare the differences among three groups. Age, gender, and education were imported as covariates in statistical analysis to avoid any confounding effects. Multiple comparisons in $\mathrm{ReHo} / \mathrm{CBF}$ ratio analysis were corrected using the clusterlevel false discovery rate (FDR) method with a threshold of $P<0.05$ after the initial cluster-forming threshold corresponding to $P<0.001$. The same methods were applied to the ReHo and $\mathrm{CBF}$ data separately to detect significant differences among groups. The mean value of the $\mathrm{ReHo} / \mathrm{CBF}$ ratio in each significant cluster was extracted and used for region of interest (ROI)based analysis. The partial correlation analysis controlled for age, gender, and education was performed to find the relationships between the ReHo/CBF ratio values of significant ROIs and the neuropsychological assessments. $P<0.05$ was set for statistical significance. Bonferroni correction for multiple testing was used to adjust the $P$ values $\left(P_{c}<0.001\right)$.

\section{RESULTS}

\section{Demographics and Cognitive Characteristics}

The demographics and WMH volumes are presented in Table $\mathbf{1 .}$ Compared with the SIVD-NCI group and NC group, the SIVDMCI group showed a significantly higher WMH burden. The levels of systolic blood pressure and the total cholesterol in patient groups are significantly different from those in the NC group. No significant differences were found in age, gender, education, triglycerides levels, and diastolic blood pressure levels.

Table 2 presents the results of the neuropsychological assessments. The results of the SIVD-MCI group were significantly worse than those of the other two groups. When compared with the NC group, the SIVD-NCI group performed significantly worse on the TMT-B test, immediate recall test, and delay recall test of AVLT.

\section{Global ReHo-CBF Coupling Analysis}

Significantly across-voxel correlation coefficients between ReHo and $\mathrm{CBF}$ were found for each participant. Compared with
TABLE 2 | Cognitive characteristics of the participants.

\begin{tabular}{lcccc}
\hline & NC & SIVD-NCI & SIVD-MCl & $P$ value \\
\hline MMSE & $28.00 \pm 1.06$ & $27.85 \pm 0.92$ & $23.93 \pm 1.90^{\mathrm{a}, \mathrm{b}}$ & $<0.001$ \\
CDR & 0 & 0 & 0.5 & $/$ \\
ADL & $20.03 \pm 0.66$ & $21.22 \pm 0.81$ & $23.87 \pm 0.35^{\mathrm{a}, \mathrm{b}}$ & $<0.001$ \\
HAMD & $2.45 \pm 1.93$ & $2.56 \pm 1.49$ & $2.46 \pm 1.37$ & 0.719 \\
AVLT & & & & \\
Immediate & $9.54 \pm 2.12$ & $7.61 \pm 2.73^{\mathrm{a}}$ & $4.82 \pm 2.53^{\mathrm{a}, \mathrm{b}}$ & $<0.001$ \\
recall & & & & \\
Delay recall & $8.83 \pm 2.33$ & $6.96 \pm 3.05^{\mathrm{a}}$ & $4.00 \pm 2.69^{\mathrm{a}, \mathrm{b}}$ & $<0.001$ \\
Recognition & $27.58 \pm 1.67$ & $27.50 \pm 1.18$ & $23.61 \pm 4.45^{\mathrm{a}, \mathrm{b}}$ & $<0.001$ \\
BNT & $24.00 \pm 3.45$ & $23.96 \pm 3.36$ & $18.36 \pm 3.97^{\mathrm{a}, \mathrm{b}}$ & $<0.001$ \\
CDT & $3.16 \pm 0.70$ & $3.46 \pm 0.65$ & $2.46 \pm 1.10^{\mathrm{a}, \mathrm{b}}$ & $<0.001$ \\
TMT-A (s) & $79.67 \pm 25.72$ & $104.30 \pm 42.56$ & $170.40 \pm 72.03^{\mathrm{a}, \mathrm{b}}$ & $<0.001$ \\
TMT-B (s) & $184.80 \pm 80.33$ & $265.12 \pm 92.49^{\mathrm{a}}$ & $391.00 \pm 112.47^{\mathrm{a}, \mathrm{b}}$ & $<0.001$ \\
Stroop-1 test & $107.87 \pm 4.57$ & $104.84 \pm 6.96$ & $81.94 \pm 20.09^{\mathrm{a}, \mathrm{b}}$ & $<0.001$ \\
Stroop-2 test & $99.91 \pm 11.62$ & $91.50 \pm 13.68$ & $67.57 \pm 24.59^{\mathrm{a}, \mathrm{b}}$ & $<0.001$ \\
\hline
\end{tabular}

a Significant compared to the NC group $(P<0.05)$.

${ }^{b}$ Significant compared to the SIVD-NCI group $(P<0.05)$.

MMSE, Mini-Mental State Examination; HAMD, Hamilton Depression Rating Scale; AVLT, Auditory Verbal Learning Test; BNT, Boston Naming Test; CDT, Clock Drawing Test; TMT, Trail Making Test.

NC, patients with SIVD showed a downward trend of ReHoCBF coupling. The SIVD-MCI group had significantly more obvious ReHo-CBF decoupling than the NC group $(P=0.004)$ (Figure 1A). Three representative correlation maps of a $\mathrm{NC}$ participant, a SIVD-NCI patient and a SIVD-MCI patient are shown in Figure 1B. The NC showed a positive correlation between ReHo and CBF (blue; $r=0.24, P<0.001$ ), while the interaction was weaker in a SIVD-NCI patient (red; $r=0.06$, $P<0.001)$. The ReHo values were inversely proportional to the CBF of a SIVD-MCI patient (orange; $r=-0.12$, $P<0.001)$.

\section{ReHo/CBF Ratio Analysis}

Brain regions with significantly altered $\mathrm{ReHo} / \mathrm{CBF}$ ratios controlled for age, gender, and education $(P<0.05$, clusterlevel FDR corrected, cluster-forming threshold at voxel-level $P<0.001$ ) were found in the left insula (INS.L), right

TABLE 1 | Demographic and clinical characteristics of the participants.

\begin{tabular}{lccc}
\hline & NC(N = 24) & SIVD-NCI $(\mathbf{N}=\mathbf{2 6})$ & SIVD-MCI (N=28) \\
\hline Gender (M/F) & $10 / 14$ & $16 / 10$ & $16 / 12$ \\
Age (year) & $68.43 \pm 8.02$ & $70.33 \pm 3.83$ & $70.73 \pm 5.58$ \\
Education (year) & $10.50 \pm 2.59$ & $10.37 \pm 2.25$ & $9.35 \pm 1.68$ \\
Systolic BP (mmHg) & $132.66 \pm 16.61$ & $143.23 \pm 22.32^{\mathrm{a}}$ & $149.79 \pm 24.26^{\mathrm{a}}$ \\
Diastolic BP (mmHg) & $80.37 \pm 11.88$ & $80.80 \pm 11.84$ & $79.37 \pm 16.44$ \\
Triglycerides (mmol/L) & $1.67 \pm 1.91$ & $1.43 \pm 0.50$ & 0.068 \\
Total cholesterol (mmol/L) & $5.40 \pm 1.26$ & $4.89 \pm 1.39^{\mathrm{a}}$ & 0.117 \\
WMH volume (ml) & $1.46 \pm 0.70$ & $12.61 \pm 5.0^{\mathrm{a}}$ & 0.71 \\
\hline
\end{tabular}

The data are presented as the means $\pm S D$ s. WMH, white matter hyperintensity.

a Significant compared to the NC group, $P<0.05$.

${ }^{b}$ Significant compared to the SIVD-NCl group, $P<0.05$. 


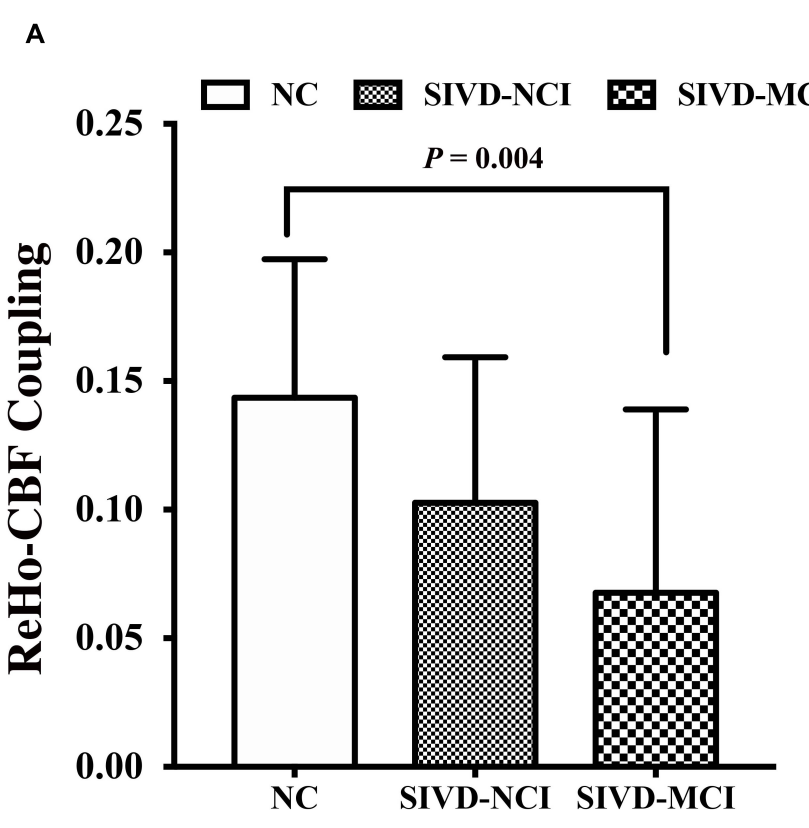

B
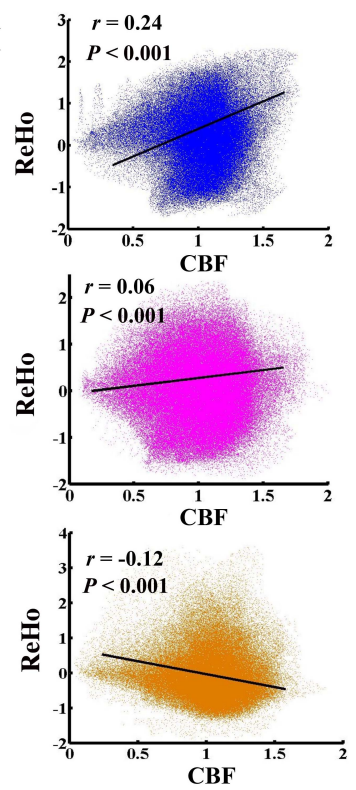

FIGURE 1 | (A) Global ReHo-CBF coupling changes among three groups; SIVD-MCl group shows a significantly reduced ReHo-CBF coupling compared with NC group $(P=0.004)$. (B) Scatter plots of spatial correlation across voxels between ReHo and CBF of a normal control (blue; $r=0.24, P<0.001$ ); a SIVD-NCl patient (red; $r=0.06, P<0.001$ ) and a SIVD-MCl patient (orange; $r=-0.12, P<0.001$ ).

middle temporal gyrus (MTG.R), right precuneus (PCUN.R), left precentral gyrus (PreCG.L), and left inferior parietal lobule but not the supramarginal and angular gyri (IPL.L) (Figure 2 and Table 3). We also detected significant differences in the values of ReHo and CBF separately. These differences are presented in Table 3.

Figure 3 illustrates the post hoc results of the ReHo/CBF ratios between each pair of the groups. The $\mathrm{ReHo} / \mathrm{CBF}$ ratios in the INS.L, PreCG.L and IPL.L of SIVD-MCI group and the ratios in the MTG.R of the SIVD-NCI group were significantly lower than those in the NC group. Compared with the SIVD-NCI group, the SIVD-MCI group showed a significantly decreased ReHo/CBF ratio at the PreCG.L. In addition, the increased ratio was found in the PCUN.R when compared between the SIVD-MCI group and the NC group $(P<0.05)$.

\section{Correlation Analysis}

We found that the mean values of ReHo/CBF ratio at the PreCG.L in SIVD-NCI group exhibited a positive correlation with MMSE scores $(r=0.55, P=0.006)$. However, the correlation did not persist after Bonferroni correction $\left(P_{c}<0.001\right)$.

\section{DISCUSSION}

In the current study, we evaluated the neurovascular coupling changes in SIVD patients by combining BOLD and ASL techniques. Although $\mathrm{CBF}$ was significantly correlated with ReHo in three groups, the SIVD patients had reduced global ReHo-CBF coupling relative to the NC group. Significantly altered $\mathrm{ReHo} / \mathrm{CBF}$ ratios were found in the left insula, right middle temporal gyrus, right precuneus, left precentral gyrus, and left inferior parietal lobule but not the supramarginal and angular gyri. The ratio in the right middle temporal gyrus of the SIVD-NCI group was significantly different from that in the NC group. Additionally, we also found $\mathrm{ReHo} / \mathrm{CBF}$ ratio deficits in the PreCG.L of the SIVD-NCI group were significantly correlated with the MMSE scores. These findings may improve our understanding about the neural mechanisms of SIVD from the perspective of dysfunction of the NVU.

It is well known that the NVU is the foundation of brain physiological activities based on neurons, astrocytes, smooth muscle cells, and endothelial cells (Nicolakakis et al., 2008). Coinciding with previous studies (Zhu et al., 2017; Guo et al., 2019), we found a significant correlation between CBF and ReHo in NC which represented normal neurovascular coupling that depends on the integrity of the NVU. Previous research has suggested that the endothelial cell damage could be an early pathological basis of neural injury in cerebral vascular disease (Wong et al., 2019). Injuries to the NVU could be the crucial point to cerebral vascular disease, but the mechanisms remain obscure (Murphy et al., 2016). In the present study, we found a weaker global ReHo-CBF coupling in a SIVDNCI patient than that of a normal participant. Moreover, an inversed trend of coupling was found in a SIVD-MCI patient (Figure 1A). The results at the group level also showed decoupling of $\mathrm{CBF}$ and $\mathrm{ReHo}$, and it may possibly illustrate that SIVD may weaken the connection between neuronal activities and the vascular responses and thus lead to dysfunction of the NVU (Figure 1B). 


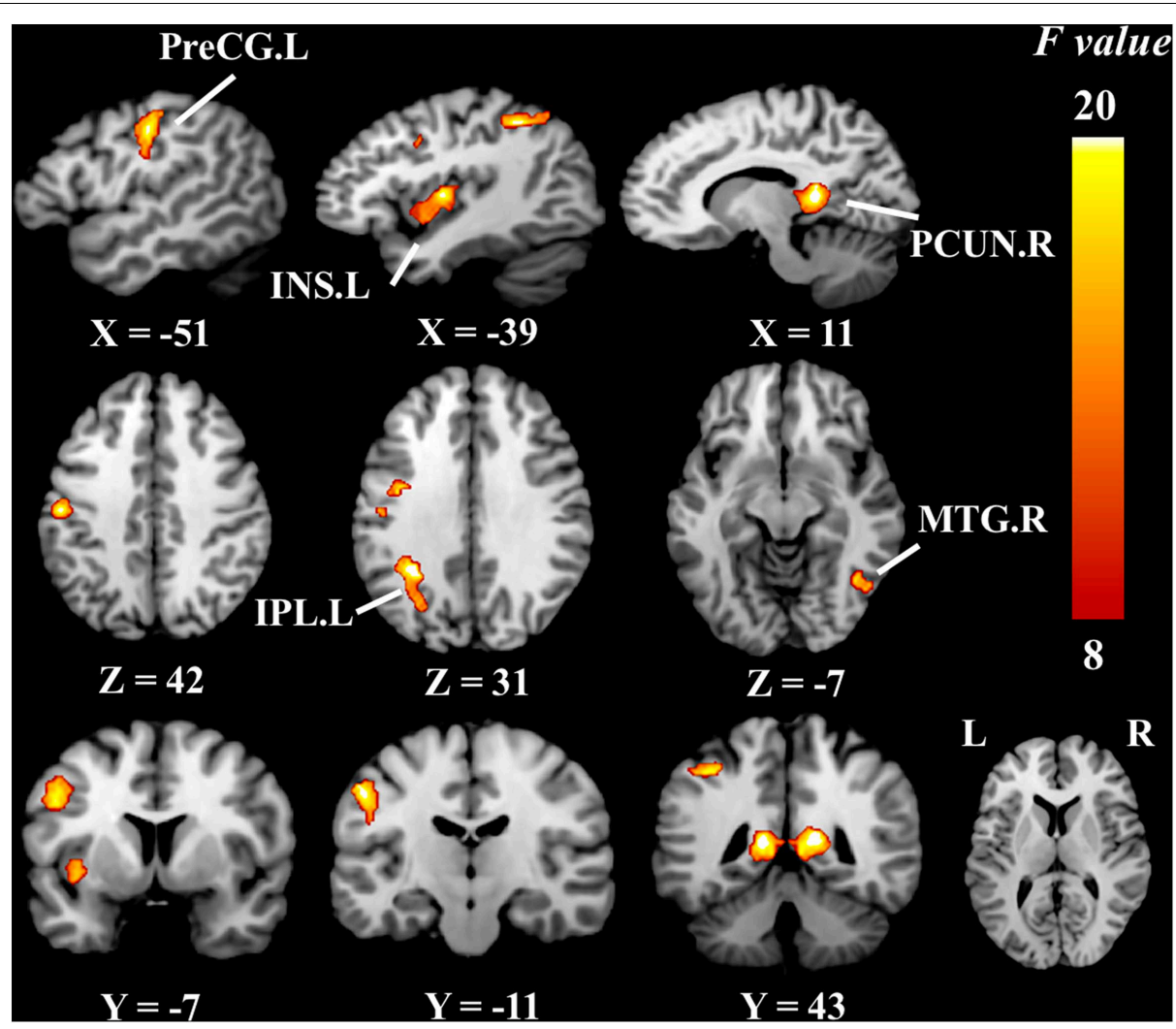

FIGURE 2 | Brain regions of group differences in ReHo/CBF ratio values controlling for age gender and education (cluster-level $P<0.05$, FDR corrected, cluster-forming threshold at voxel-level $P<0.001$ ); $x, y, z$, coordinates of primary peak locations in MNI space; INS, insula; MTG, middle temporal gyrus; PCUN, precuneus; PreCG, precentral gyrus; IPL, inferior parietal, but supramarginal and angular gyri; L, left; R, right. The color bar represents the $F$ values.

TABLE 3 | Group differences in the value of the ReHo/CBF ratio.

\begin{tabular}{|c|c|c|c|c|c|c|c|c|}
\hline \multirow[t]{2}{*}{ Brain region } & \multicolumn{3}{|c|}{ MNI coordinates (mm) } & \multirow[t]{2}{*}{ Voxels } & \multirow[t]{2}{*}{$F$ value } & \multicolumn{3}{|c|}{ Peak $T$ value } \\
\hline & $x$ & $y$ & $z$ & & & SIVD-NCI vs. NC & SIVD-MCI vs. NC & SIVD-MCI vs. SIVD-NCI \\
\hline INS.L & -36 & -10 & 6 & 256 & 15.63 & & $-5.59^{a}$ & \\
\hline MTG.R & 64 & -60 & 8 & 185 & 11.91 & -4.67 & & \\
\hline PCUN.R & 10 & -40 & 6 & 599 & 18.64 & & $5.95^{a, b}$ & \\
\hline PreCG.L & -54 & -14 & 44 & 599 & 16.28 & & $-5.03^{a}$ & -5.38 \\
\hline IPL.L & -32 & -48 & 36 & 245 & 16.26 & & $-5.53^{a}$ & \\
\hline
\end{tabular}

MNI, Montreal Neurological Institute; $x, y, z$, coordinates of primary peak locations in MNI space; INS, insula; MTG, middle temporal gyrus; PCUN, precuneus; PreCG,

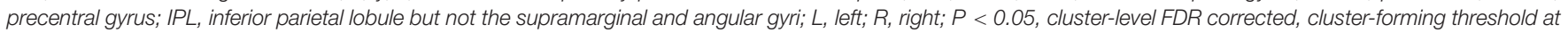
voxel-level $P<0.001$.

a Statistical differences in the value of $\mathrm{ReH}$.

${ }^{b}$ Statistical differences in the value of $\mathrm{CBF}$.

Compared to the global ReHo-CBF correlation coefficient, which describes the comprehensive changes of neurovascular coupling in the whole brain, the $\mathrm{ReHo} / \mathrm{CBF}$ ratio could give more specific information about the alterations of NVU in local brain regions. In SIVD, endothelial distress in cerebral small vessels can potentiate flow dysregulation, chronic hypoxia, and stimulated inflammation in different brain areas (Arba et al., 2018; Moretti and Caruso, 2020). Hypoperfusion can further reduce the activities and damage the structure of microglia, astrocytes, and pericytes. Moreover, neural disorders can in turn affect the $\mathrm{CBF}$ and finally result in the dysfunction of NVU (Wardlaw et al., 2019).

Previous studies have confirmed that injuries to cerebrovascular endothelial cells and blood-brain barrier are essential for small-vessel disease (Nation et al., 2019; Wong et al., 2019). Destructions of the structural integrity of nerve fibers and abnormal FC of neurons in SIVD have been demonstrated as reliable biomarkers of human cognitive dysfunction (Liu et al., 2019a,b). However, a single index cannot reflect the interactions between the neuronal activity and cerebral perfusion, so the 


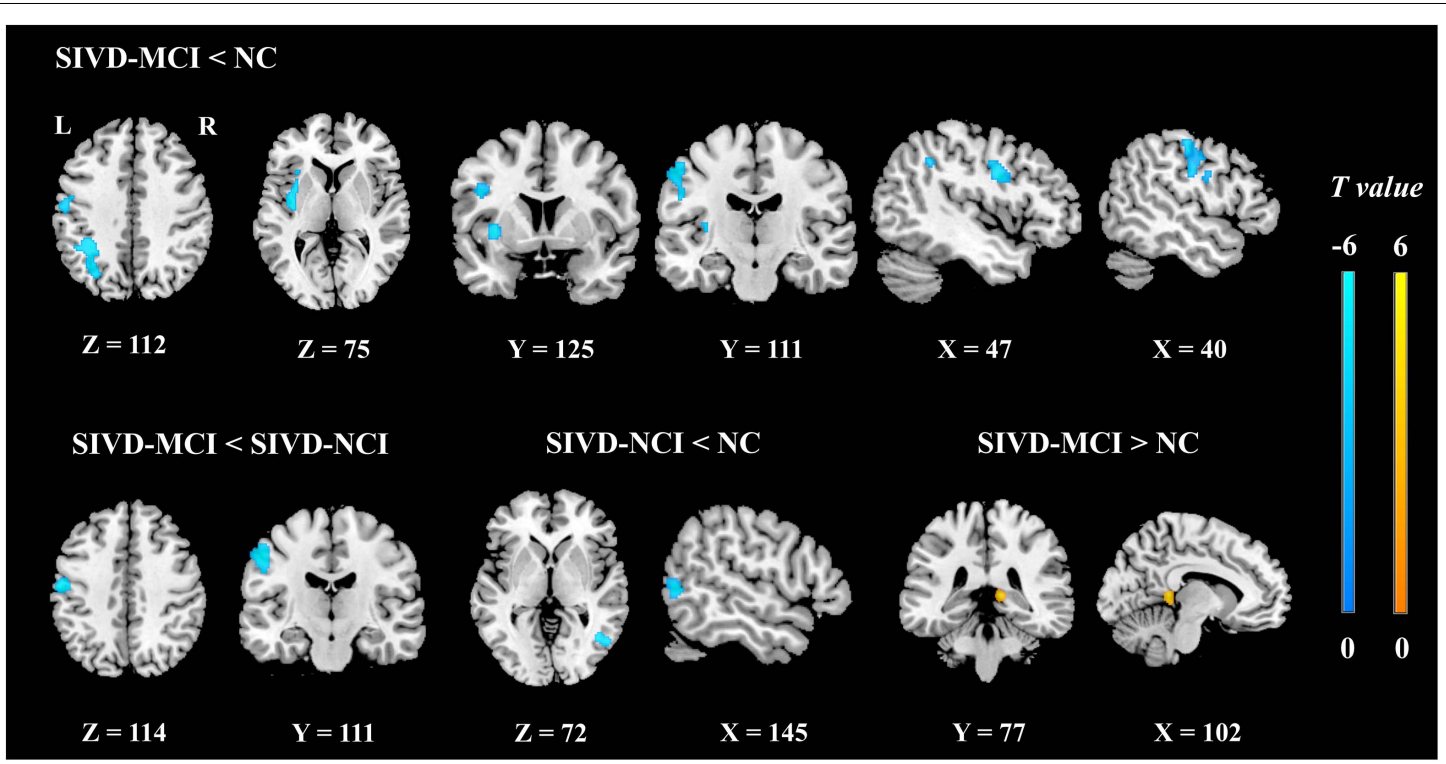

FIGURE 3 | The post hoc analyses of ReHo/CBF ratio values between each pair of groups controlling for age, gender, and education (cluster-level $P<0.05$, FDR corrected, cluster-forming threshold at voxel-level $P<0.001) ; x, y, z$, coordinates of primary peak locations in the MNI space. The color bars represent the $T$ values.

$\mathrm{ReHo} / \mathrm{CBF}$ ratio may be used as a comprehensive marker to evaluate the NVU function. The $\mathrm{ReHo} / \mathrm{CBF}$ ratio remains balanced in healthy brains, and deviation from this balance may result in either an increased or decreased $\mathrm{ReHo} / \mathrm{CBF}$ ratio. We found that $\mathrm{ReHo} / \mathrm{CBF}$ ratios in the left insula, left precentral gyrus, and left inferior parietal lobule but not the supramarginal and angular gyri of the SIVD-MCI group were significantly decreased, while the $\mathrm{ReHo} / \mathrm{CBF}$ ratio in the right precuneus was increased with respect to those of the NC group. We also obtained the same statistical results by evaluating the ReHo and CBF values, respectively (Table 3 ). The decreased ratios may indicate reduced strength between neurons and the surrounding brain areas supplied by a unit of CBF, whereas explaining the increased ratio is slightly complicated because the ReHo and $\mathrm{CBF}$ values were both increased in the right precuneus. This may indicate that disorders of the NVU lead to abnormally enhanced functional connections supplied by redundant blood flow.

More importantly, we found that the $\mathrm{ReHo} / \mathrm{CBF}$ ratio was significantly decreased in the right middle temporal gyrus of the SIVD-NCI group with respect to the NC group, and the $\mathrm{ReHo} / \mathrm{CBF}$ ratio was also found significantly decreased in the left precentral gyrus of the SIVD-MCI group with respect to the SIVD-NCI group. However, the results did not show the significant intergroup differences in ReHo and CBF, respectively. This may indicate that the $\mathrm{ReHo} / \mathrm{CBF}$ ratio could be more sensitive for detecting neurovascular coupling changes at the early stage of the disease and during its progression than the separate ReHo and CBF. In sum, CBF, ReHo, and the ReHo/CBF ratio could give comprehensive information and should be assessed jointly to explore pathological changes in SIVD.

In this study, we identified the altered neurovascular coupling in several brain regions associated with cognitive impairment. The SIVD-NCI patients showed a significantly decreased
$\mathrm{ReHo} / \mathrm{CBF}$ ratio in the left precentral gyrus, which was positively correlated with the MMSE scores. It is acknowledged that the precentral gyrus is the core brain region of the sensorimotor network and is mainly involved in cognitive control and motor activities (Park and Friston, 2013). This finding is consistent with the previous studies showing that the precentral gyrus is susceptible to cerebral vascular diseases, which may lead to cognitive injuries (Vipin et al., 2018; Caruso et al., 2019). Decreased ReHo/CBF ratios were also found in the insula, inferior parietal lobule, and middle temporal gyrus, which are regarded as the seats of specific cognitive domains. For example, the insula has been found involved in language processing (Gogolla, 2017), and hypoperfusion of this area is related to the degree of cognitive impairment (Sun et al., 2016). In our study, we found that SIVD patients performed worse in the Boston Naming Test than NC, which represented language function deficits. The results echo the previous study. The parietal regions and medial temporal regions have been suggested to be related to visuospatial attention and working memory (Wang et al., 2015; Lim et al., 2020). The lower scores in the Trial-Making Test and Stroop Test of SIVD patients in the present study may demonstrate that the abnormally decreased $\mathrm{ReHo} / \mathrm{CBF}$ ratios in these brain regions were correlated with deficits in these specific cognitive domains. Unexpectedly, a significantly increased ReHo, CBF, and $\mathrm{ReHo} / \mathrm{CBF}$ ratio were found in the precuneus, a pivotal node in regulating cognitive functions (van Leijsen et al., 2019). Abnormal deviation and activation of neurovascular coupling in the precuneus may be treated as a sensitive marker to reflect disturbances of the NVU associated with cognitive impairment.

The study has several limitations. Firstly, it is a relatively limited sample size research, and the severity of the disease was confined to the early stage. Patients with vascular dementia were not included. Future longitudinal studies with a larger group 
and dementia subjects are recommended. Secondly, technical limitations may have reduced the precision in the calculation of ReHo and CBF. Therefore, future studies with advanced methods and scanning parameters are recommended to strengthen the accuracy of the results. Thirdly, the correlation results between the $\mathrm{ReHo} / \mathrm{CBF}$ ratio in the left precentral gyrus and the MMSE scores were not valid after Bonferroni correction. Thus, our findings should be regarded as an exploratory analysis. Finally, although the ReHo-CBF correlation and $\mathrm{ReHo} / \mathrm{CBF}$ ratio are recommended for studying neurovascular coupling, they are both indirect measures for vascular response and neuronal activity. The ReHo-CBF correlation and $\mathrm{ReHo} / \mathrm{CBF}$ ratio may not be sufficiently precise to truly reflect neurovascular coupling. This study has clarified a vital role of studying neurovascular coupling to understand the mechanisms of SIVD-related cognitive influence. Our research may provide a new particular imaging marker for deeply exploring the pathology of SIVD.

\section{CONCLUSION}

In conclusion, we revealed a disrupted coupling between cerebral perfusion and functional activities of SIVD by jointly applying the BOLD and ASL techniques. Patients with SIVD show dysregulated neurovascular coupling that associate with disease severity and cognitive impairment. The findings may provide a new field in studying the neuropathological underpinning of the disease.

\section{DATA AVAILABILITY STATEMENT}

The raw data supporting the conclusions of this article will be made available by the authors, without undue reservation.

\section{REFERENCES}

Arba, F., Giannini, A., Piccardi, B., Biagini, S., Palumbo, V., Giusti, B., et al. (2018). Small vessel disease and biomarkers of endothelial dysfunction after ischemic stroke. Eur. Stroke J. 4, 119-126. doi: 10.1177/23969873188 05905

Caruso, P., Signori, R., and Moretti, R. (2019). Small vessel disease to subcortical dementia: a dynamic model, which interfaces aging, cholinergic dysregulation and the neurovascular unit. Vasc. Health Risk Manag. 7, 259-281. doi: 10.2147/ VHRM.S190470

Diciotti, S., Orsolini, S., Salvadori, E., Giorgio, A., Toschi, N., Ciulli, S., et al. (2017). Resting state fMRI regional homogeneity correlates with cognition measures in subcortical vascular cognitive impairment. J. Neurol. Sci. 373, 1-6. doi: 10.1016/j.jns.2016.12.003

Fox, M. D., and Raichle, M. E. (2007). Spontaneous fluctuations in brain activity observed with functional magnetic resonance imaging. Nat. Rev. Neurosci. 8, 700-711. doi: 10.1038/nrn2201

Girouard, H., and Iadecola, C. (2006). Neurovascular coupling in the normal brain and in hypertension, stroke, and Alzheimer disease. J. Appl. Physiol. 100, 328-335. doi: 101152/ japplphysiol.00966.2005

Gogolla, N. (2017). The insular cortex. Curr. Biol. 27, 580-586. doi: 10.1016/j.cub. 2017.05.010

Golay, X., Hendrikse, J., and Lim, T. C. (2004). Perfusion imaging using arterial spin labeling. Top. Magn. Reson. Imaging 15, 10-27. doi: 10.1097/00002142200402000-00003

\section{ETHICS STATEMENT}

The studies involving human participants were reviewed and approved by the Ethics Committee of The First Affiliated Hospital of Chongqing Medical University. The patients/participants provided their written informed consent to participate in this study.

\section{AUTHOR CONTRIBUTIONS}

TL, XL, and LC made substantial contributions to the conception and design of the study. XL, RC, and JG made substantial contributions to the acquisition of data. XL and LC made substantial contributions to the analysis of data. XL, XL, FL, and LC contributed to the interpretations of data. XL drafted the first version of the manuscript. All the authors revised the draft for intellectual content, gave their final approval of the final version for publication, and agreed to be accountable for all aspects of the work in ensuring that questions related to the accuracy or integrity of any part of the study are appropriately investigated and resolved.

\section{FUNDING}

This work was supported by grants from the National Natural Science Foundation of China (81671666).

\section{ACKNOWLEDGMENTS}

We would like to thank all the participants for their helpful comments on this article.

Guo, X., Zhu, J., Zhang, N., Zhang, L., Qi, Y., Cai, H., et al. (2019). Altered neurovascular coupling in neuromyelitis optica. Hum. Brain Mapp. 40, 976986. doi: 10.1002/hbm.24426

Helman, A. M., and Murphy, M. P. (2016). Vascular cognitive impairment: modeling a critical neurologic disease in vitro and in vivo. Biochim. Biophys. Acta 1862, 975-982. doi: 10.1016/j.bbadis.2015.12.009

Hirano, S., and Yamada, M. (2013). Network analyses in neuroimaging studies. Brain Nerve 65, 659-667.

Hu, B., Yan, L. F., Sun, Q., Yu, Y., Zhang, J., Dai, Y. J., et al. (2019). Disturbed neurovascular coupling in type 2 diabetes mellitus patients: evidence from a comprehensive fMRI analysis. Neuroimage Clin. 22:101802. doi: 10.1016/j.nicl. 2019.101802

Li, C., Liu, C., Yin, X., Yang, J., Gui, L., Wei, L., et al. (2014). Frequency-dependent changes in the amplitude of low-frequency fluctuations in subcortical ischemic vascular disease (SIVD): a resting-state fMRI study. Behav. Brain Res. 274, 205-210. doi: 10.1016/j.bbr.2014.08.019

Liang, X., Zou, Q., He, Y., and Yang, Y. (2013). Coupling of functional connectivity and regional cerebral blood flow reveals a physiological basis for network hubs of the human brain. Proc. Natl. Acad. Sci. U.S.A. 110, 1929-1934. doi: 10.1073/ pnas. 1214900110

Lim, J. S., Kwon, H. M., and Lee, Y. S. (2020). Effect of cholinergic pathway disruption on cortical and subcortical volumes in subcortical vascular cognitive impairment. Eur. J. Neurol. 27, 210-212. doi: 10.1111/ene.14073

Liu, J., Qin, W., Wang, H., Zhang, J., Xue, R., Zhang, X., et al. (2014). Altered spontaneous activity in the default-mode network and cognitive decline in 
chronic subcortical stroke. J. Neurol. Sci. 347, 193-198. doi: 10.1016/j.jns.2014. 08.049

Liu, X., Chen, L., Cheng, R., Luo, T., Lv, F., Fang, W., et al. (2019a). Altered functional connectivity in patients with subcortical ischemic vascular disease: a resting-state fMRI study. Brain Res. 1715, 126-133. doi: 10.1016/j.brainres. 2019.03.022

Liu, X., Cheng, R., Chen, L., Luo, T., Lv, F., Gong, J., et al. (2019b). Alterations of white matter integrity in subcortical ischemic vascular disease with and without cognitive impairment: a TBSS study. J. Mol. Neurosci. 67, 595-603. doi: 10.1007/s12031-019-01266-3

Ma, J., Liu, F., Yang, B., Xue, K., Wang, P., Zhou, J., et al. (2020). Selective aberrant functional-structural coupling of multiscale brain networks in subcortical vascular mild cognitive impairment. Neurosci. Bull. 37, 287-297. doi: 10.1007/ s12264-020-00580-w

Moretti, R., and Caruso, P. (2020). Small vessel disease-related dementia: an invalid neurovascular coupling? Int. J. Mol. Sci. 21:1095. doi: 10.3390/ijms21031095

Muoio, V., Persson, P. B., and Sendeski, M. M. (2014). The neurovascular unitconcept review. Acta Physiol. 210, 790-798. doi: 10.1111/apha.12250

Murphy, M. P., Corriveau, R. A., and Wilcock, D. M. (2016). Vascular contributions to cognitive impairment and dementia (VCID). Biochim. Biophys. Acta 1862, 857-859. doi: 10.1016/j.bbadis

Nation, D. A., Sweeney, M. D., Montagne, A., Sagare, A. P., D’Orazio, L. M., Pachicano, M., et al. (2019). Blood-brain barrier breakdown is an early biomarker of human cognitive dysfunction. Nat. Med. 25, 270-276. doi: 10 . 1038/s41591-018-0297-y

Nicolakakis, N., Aboulkassim, T., Ongali, B., Lecrux, C., Fernandes, P., RosaNeto, P., et al. (2008). Complete rescue of cerebrovascular function in aged Alzheimer's disease transgenic mice by antioxidants and pioglitazone, a peroxisome proliferator-activated receptor gamma agonist. J. Neurosci. 28, 9287-9296. doi: 10.1523/JNEUROSCI

Park, H. J., and Friston, K. (2013). Structural and functional brain networks: from connections to cognition. Science 342:1238411. doi: 10.1126/science.1238411

Pasley, B., and Freeman, R. (2008). Neurovascular coupling. Scholarpedia 3:5340. doi: 10.4249 /scholarpedia.5340

Qin, Y., Zhu, W., Liu, C., Wang, Z., and Zhu, W. (2019). Functional brain connectome and its relation to mild cognitive impairment in cerebral small vessel disease patients with thalamus lacunas: a cross-sectional study. Medicine 98:e17127. doi: 10.1097/MD.0000000000017127

Reijmer, Y. D., Fotiadis, P., Piantoni, G., Boulouis, G., Kelly, K. E., Gurol, M. E., et al. (2016). Small vessel disease and cognitive impairment: the relevance of central network connections. Hum. Brain Mapp. 37, 2446-2454. doi: 10.1002/ hbm.23186

Rosenberg, G. A., Bjerke, M., and Wallin, A. (2014). Multimodal markers of inflammation in the subcortical ischemic vascular disease type of vascular cognitive impairment. Stroke 45, 1531-1538. doi: 10.1161/STROKEAHA.113. 004534

Sachdev, P., Kalaria, R., O’Brien, J., Skoog, I., Alladi, S., Black, S. E., et al. (2014). Diagnostic criteria for vascular cognitive disorders: a VASCOG statement. Alzheimer Dis. Assoc. Disord. 28, 206-218. doi: 10.1097/WAD. 0000000000000034

Shabir, O., Berwick, J., and Francis, S. E. (2018). Neurovascular dysfunction in vascular dementia, Alzheimer's and atherosclerosis. BMC Neurosci. 19:62. doi: 10.1186/s12868-018-0465-5

Sheng, J., Shen, Y., Qin, Y., Zhang, L., Jiang, B., Li, Y., et al. (2018). Spatiotemporal, metabolic, and therapeutic characterization of altered functional connectivity in major depressive disorder. Hum. Brain Mapp. 39, 1957-1971.

Stickland, R., Allen, M., Magazzini, L., Singh, K. D., Wise, R. G., and Tomassini, V. (2019). Neurovascular coupling during visual stimulation in multiple sclerosis: a MEG-fMRI study. Neuroscience 403, 54-69. doi: 10.1016/j.neuroscience. 2018. 03.018

Sun, Y., Cao, W., Ding, W., Wang, Y., Han, X., Zhou, Y., et al. (2016). Cerebral blood flow alterations as assessed by 3D ASL in cognitive impairment in patients with subcortical vascular cognitive impairment: a marker for disease severity. Front. Aging Neurosci. 8:211. doi: 10.3389/fnagi.2016.00211
Thrippleton, M. J., Backes, W. H., Sourbron, S., Ingrisch, M., van Osch, M. J. P., Dichgans, M., et al. (2019). Quantifying blood-brain barrier leakage in small vessel disease: review and consensus recommendations. Alzheimers Dement. 15, 840-858. doi: 10.1016/j.jalz.2019.01.013

Vaishnavi, S. N., Vlassenko, A. G., Rundle, M. M., Snyder, A. Z., Mintun, M. A., and Raichle, M. E. (2010). Regional aerobic glycolysis in the human brain. Proc. Natl. Acad. Sci. U.S.A. 107, 17757-17762. doi: 10.1073/pnas.1010459107

van, Leijsen, E. M. C., van, Uden, I. W. M., Bergkamp, M. I., van, et al. (2019). Longitudinal changes in rich club organization and cognition in cerebral small vessel disease. Neuroimage Clin. 24:102048. doi: 10.1016/j.nicl.2019. 102048

Vipin, A., Foo, H. J. L., Lim, J. K. W., Chander, R. J., Yong, T. T., Ng, A. S. L., et al. (2018). Regional white matter hyperintensity influences grey matter atrophy in mild cognitive impairment. J. Alzheimers Dis. 66, 533-549. doi: 10.3233/JAD180280

Wallin, A., Roman, G. C., Esiri, M., Kettunen, P., Svensson, J., Paraskevas, G. P., et al. (2018). Update on vascular cognitive impairment associated with subcortical small-vessel disease. J. Alzheimers Dis. 62, 1417-1441. doi: 10.3233/ JAD- 170803

Wang, J., Yang, Y., Fan, L., Xu, J., Li, C., Liu, Y., et al. (2015). Convergent functional architecture of the superior parietal lobule unraveled with multimodal neuroimaging approaches. Hum. Brain Mapp. 36, 238-257. doi: 10.1002/hbm. 22626

Wardlaw, J. M., Makin, S. J., Valdés Hernández, M. C., Armitage, P. A., Heye, A. K., Chappell, F. M., et al. (2017). Blood-brain barrier failure as a core mechanism in cerebral small vessel disease and dementia: evidence from a cohort study. Alzheimers Dement. 13, 634-643. doi: 10.1016/j.jalz.2016.09.006

Wardlaw, J. M., Smith, C., and Dichgans, M. (2019). Small vessel disease: mechanisms and clinical implications. Lancet Neurol. 18, 684-696. doi: 10.1016/ S1474-4422(19)30079-1

Wong, S. M., Jansen, J. F. A., Zhang, C. E., Hoff, E. I., Staals, J., van, et al. (2019). Blood-brain barrier impairment and hypoperfusion are linked in cerebral small vessel disease. Neurology 92, e1669-e1677. doi: 10.1212/WNL. 0000000000007263

Xu, X., Lau, K. K., Wong, Y. K., Mak, H. K. F., and Hui, E. S. (2018). The effect of the total small vessel disease burden on the structural brain network. Sci. Rep. 8:7442. doi: 10.1038/s41598-018-25917-4

Zang, Y., Jiang, T., Lu, Y., He, Y., and Tian, L. (2004). Regional homogeneity approach to fMRI data analysis. Neuroimage 22, 394-400. doi: 10.1016/j. neuroimage.2003.12.030

Zhang, C. E., Wong, S. M., Uiterwijk, R., Backes, W. H., Jansen, J. F. A., Jeukens, C., et al. (2019). Blood-brain barrier leakage in relation to white matter hyperintensity volume and cognition in small vessel disease and normal aging. Brain Imaging Behav. 13, 389-395. doi: 10.1007/s11682-018-9855-7

Zhu, J., Zhuo, C., Xu, L., Liu, F., Qin, W., and Yu, C. (2017). Altered coupling between resting-state cerebral blood flow and functional connectivity in schizophrenia. Schizophr. Bull. 43, 1363-1374. doi: 10.1093/schbul/ sbx051

Zhu, S., Fang, Z., Hu, S., Wang, Z., and Rao, H. (2013). Resting state brain function analysis using concurrent BOLD in ASL perfusion fMRI. PLoS One 8:e65884. doi: 10.1371/journal.pone.0065884

Zlokovic, B. V. (2010). Neurodegeneration and the neurovascular unit. Nat. Med. 16, 1370-1371. doi: $10.1038 / \mathrm{nm} 1210-1370$

Conflict of Interest: The authors declare that the research was conducted in the absence of any commercial or financial relationships that could be construed as a potential conflict of interest.

Copyright (C) 2021 Liu, Cheng, Chen, Gong, Luo and Lv. This is an open-access article distributed under the terms of the Creative Commons Attribution License (CC BY). The use, distribution or reproduction in other forums is permitted, provided the original author(s) and the copyright owner(s) are credited and that the original publication in this journal is cited, in accordance with accepted academic practice. No use, distribution or reproduction is permitted which does not comply with these terms. 